\title{
Molecular dynamic study of the jet noise about the micro-scale tube
}

Yong-Fei XUE ${ }^{1,2}$, Jun-Long XIE $^{1}$ Huan-Lin DUAN ${ }^{2}$, Ke-Qi WU ${ }^{1}$

\footnotetext{
${ }^{1}$ School of Energy and Power Engineering, Huazhong university of science and technology, Wuhan 430074, China e-mail: xue_yongfei@aliyun.com; hustxj1@163.com; wkqfmae@126.com

${ }^{2}$ College of Civil Engineering, Henan institute of engineering, Zhengzhou 450007, Henan, China e-mail: xue_yongfei@aliyun.com; meiwei@sohu.com.
}

\section{ABSTRACT}

This paper studies the two-dimensional micro-scale molecular simulations of the jet flow using Lagrange discrete systems and adopting Andresen flexible constraint mechanism. At the effects of different excitation conditions and boundary conditions, the low Mach number flow field and sound field are obtained, and characteristic results are given. At the jet flow and pipe flow regions, particles velocities distribution is consistent with traditional method. There are many small groups which have a bigger velocity value, and form the disturbance sources. The stronger interaction with the tube wall produces greater sound pressure, thus random collisions method at the tube wall is effective to deal with sound propagation problem. Therefore, this paper offers preliminary and calculated basis for the molecular and macroscopic quantum aerodynamic problems.

Key words: jet flow, sound propagation, constraint molecular dynamics, Lagrange density, micro scale tube

\section{INTRODUCTION}

The traditional top-down fluid numerical simulations often focus on the discrete truncation errors, but ignore the physical conservation and numerical stability. Then the microscopic discrete model can be discovered the nature of the macro phenomena profoundly, because it is bottom-up approach for understanding the macro-micro particles and fluid phenomena essentials [1]. Lattice Boltzmann method has been the most successful applications [2]. From lattice Boltzmann method to molecular level study, the main considering objects changes into very small scale, so it would be more able to reveal acoustic mechanism.

Once the atomic natures of matters are determined, quantum mechanics describes the microscopic world, the molecular composition and the microscopic behavior state of particles. Thus makes the situation become more complicated. So the molecular dynamics simulation study is widely used [프, $\underline{4}$ ]. On the other hand, due to the traditional jet flow sound caused by the traditional flow has been affected a lot of attention [] ], and these results usually adopt nanometer size, need the huge amount of computation, so the research literatures are rarely reported about jet at molecular dynamics levels. This paper would introduce the high-performance computing method, and study the low Reynolds-number jet flow fields by molecular dynamics method, to provide a sound basis for the correct understanding of the micro-scale sound spread phenomenon, to explore microscopic mechanism of the jet sound.

\section{SIMULATION MODELS AND PRINCIPLES}

The air is a mixture with oxygen and nitrogen molecules by a certain mass ratio. According to the literature values $[\underline{6}, \underline{7}]$, the energy and length parameters use the Lorentz-Berthelot mixing rule [ $\underline{8}$ ]. Its potential energy function models are the two-body Lennard-Jones potential model [3]]. The physical simulations are non-dimensional treatment [ 8 ]. The dimensional length, time, temperature, and density are as follows: 


$$
\begin{aligned}
& r^{*}=r / \sigma \\
& t^{*}=\left(t^{2} \varepsilon / m \sigma^{2}\right)^{0.5} \\
& T^{*}=k_{\mathrm{B}} T / \varepsilon \\
& \rho^{*}=\rho \sigma^{3} / m
\end{aligned}
$$

Where $r^{*}$ is dimensional length, $r$ is diameter [nm], $\sigma$ is length parameter [nm], $t^{*}$ is dimensional time; $t$ is time[s], $m$ is mass $[\mathrm{kg}], \varepsilon$ is energy parameter $[\mathrm{J}], T^{*}$ is dimensional temperature, $T$ is temperature $[\mathrm{K}], k_{\mathrm{B}}$ is Boltzmann constant, $\rho^{*}$ is dimensional density, and $\rho$ is density $\left[\mathrm{kg} / \mathrm{m}^{3}\right]$.

Analog pipeline size is 250 angstroms wide, and 1200 angstroms length. The particles at sidewall are arranged as looped and compact particle forms, named case 1 and case 2 . Outer flow field size is $3000 \times 8000$ $(1200 \times 3200)$ square angstroms. Calculation model is shown in fig. 1 , the tube wall is a solid boundary on the pipe length, and the other boundary is the gas boundary. Import is simplified as a particle gun, producing various velocities continuously. At the outlet boundary, flow is freely.

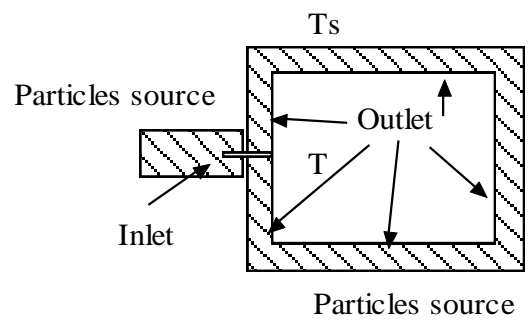

a) Physics model

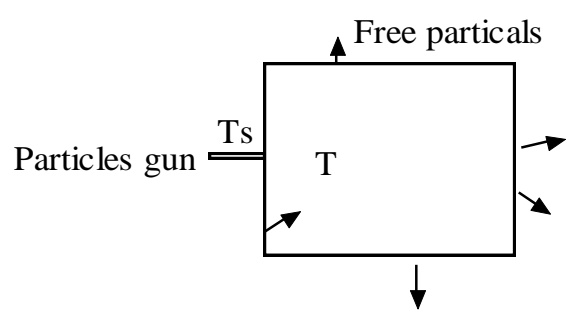

b) Simplified model

Figure 1: Calculation model

According to the ideal gas characteristic, the system macro-pressure is as below [ $\underline{3}]$ :

$$
\begin{aligned}
& -3 P V+\sum \sum r_{\mathrm{ij}} v^{\prime}\left(r_{\mathrm{ij}}\right)=-3 N k_{\mathrm{B}} T \\
& P=\left(N k_{\mathrm{B}} T+\sum \sum r_{\mathrm{ij}} f_{\mathrm{ij}} / 3\right) / V
\end{aligned}
$$

Where $P$ is pressure [Pa], $V$ is volume $\left[\mathrm{m}^{3}\right], N$ is number of molecules, $r_{\mathrm{ij}}$ is distance $[\mathrm{nm}]$ between molecule $\mathrm{i}$ and $\mathrm{j}$, and $f_{\mathrm{ij}}$ is Lennard-Jones force $[\mathrm{N}]$.

Based from the relationship between the internal energy and temperature, kinetic energy is:

$$
E_{\text {kinetic }}=\sum p_{\mathrm{i}}^{2} / 2 m_{\mathrm{i}}=0.5 k_{\mathrm{B}} T\left(3 N-N_{\mathrm{c}}\right)
$$

Where $\mathrm{E}_{\text {kinetic }}$ is kinetic energy [J], $p$ is momentum $[\mathrm{kg} \cdot \mathrm{m} / \mathrm{s}]$, and $N_{\mathrm{c}}$ is the number of molecules at equilibrium statement. So, the temperature is directly controlled by the internal energy. More, we can add flexible control on the total system temperature, and temperature control factor as below:

$$
\lambda=\left\{1+\left[\delta t\left(T_{0}-T(t)\right)\right] /[\tau T(t)]\right\}^{-0.5}
$$


Where $\lambda$ is temperature control factor, $\delta$ is space step length [nm], $\tau$ is relaxation factor, and $T_{0}$ is equilibrium temperature $[\mathrm{K}]$.

Weakened the long-range potential to bring direct truncated non-physical factors, the potential force are softened by math technology [3]. At the inlet, the velocities of particles are displayed approximate logarithmic distribution [5]. Definition of grid position is as $(x, y)$, the position of equilibrium by the disturbance and displacement is as $\eta\left(\eta_{i}, i=1,2\right.$.), the discrete macro-pneumatic system using Lagrange density of the sound field representation [9]:

$$
\left.L D=L T-L V=\left[\rho_{0}\right) \&+2 p_{0} \nabla \cdot \eta-\gamma p_{0}(\nabla \cdot \eta)^{2}\right] / 2
$$

Where $L D$ is Lagrange density $\left[\mathrm{kg} / \mathrm{m}^{3}\right], L T$ is kinetic density $\left[\mathrm{kg} / \mathrm{m}^{3}\right], L V$ is potential density $\left[\mathrm{kg} / \mathrm{m}^{3}\right]$, the subscript tag 0 is equilibrium statement, $\nabla$ is divergence sign, and $\eta$ is displacement [nm].

The initial velocity distribution is according to Maxwell random values, and its' initial acceleration are set to zero. At the different time step, the speed values at the import and outlet boundary are reassigned, but the overall region adopts the direct control method in the case 1, and Andresen temperature control in the case 2. The calculated program uses dynamic target arrays to update the number of particles, and pass them pointer arrays to update its coordinate's values and the others (as in fig. 2). If the particles is beyond the control region, then force them freezing, that is stationary. Time step length is taken as 0.0072 , and the total number of time steps is $10^{+5}$. The front steps are for fully developed flow, when the flow lines show cyclical changes, and meet requirements of the state statistical system. The subsequent steps are for the relevant parameters statistics, such as the sound pressure level (SPL $[\mathrm{dB}])$.

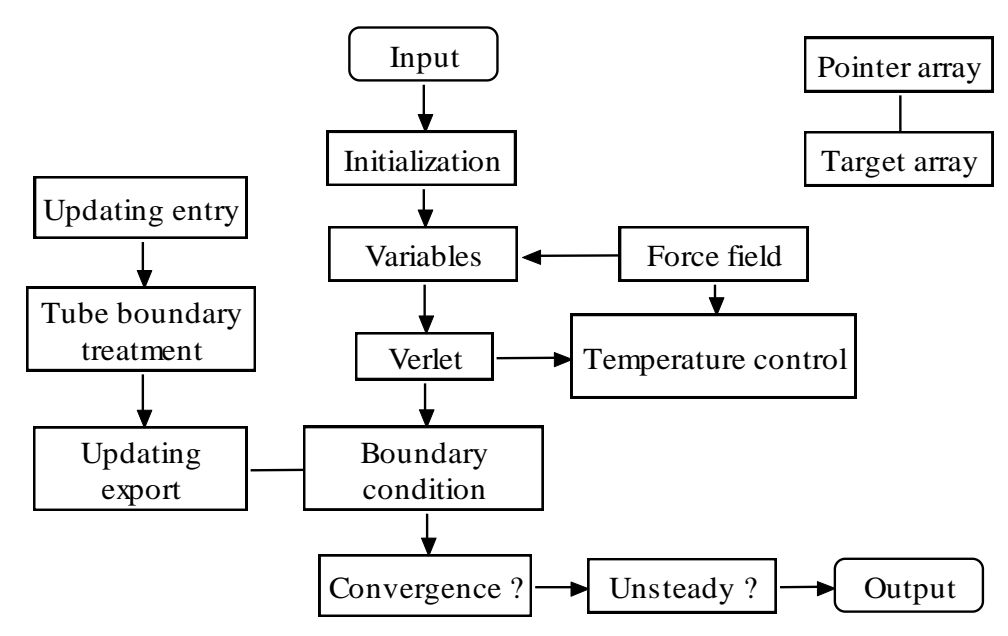

Figure 2: Program chart

Considering the physical mechanisms, system pressures are directly related to the definition style, we consider the following four options about Lagrange density: the direct calculation of sound pressure is arranged as option 1, which is depended on macro parameter (density and pressure). Local parameters are arranged as option 2, mixed parameters are arranged as option 3, and the traditional definition is option 4.

\section{FURTHER ANALYSIS AND DISCUSSION}

Numerical experiments are carried out firstly in the tube pipeline. With a random velocity along the vertical direction of the pipe diameter, the results are compared to conventional macro theory consistently. Although the Reynolds number is small, but the molecular layer flow speed still exist a big difference, showing the nature of turbulence. The existence of the Y velocity shows random features. The viscosity values $\left[\mathrm{m}^{2} / \mathrm{s}\right]$ are smaller in center region, but bigger at wall aside (as in fig. 3 ). 
Near-field velocity pulse is relative to the mainstream pulse. The $\mathrm{X}$ acceleration value is fluctuating around zero. At central tube, the axial velocity fluctuation is significantly. The $\mathrm{Y}$ acceleration value displays small fluctuations, a few larger speed particles clusters still exist. Far-field acceleration value is almost zero. The Y velocity distribution at $10 d$ place from nozzles is normal (compared with the traditional distribution), but still with small fluctuations (as in fig. 4). Higher speed flow particle clusters exist, and forms the disturbance source. At the both sides of axis, the velocity distribution changes largely by a few big-speed particles, the overall is symmetry. The negative velocities display back flow and entrainment.

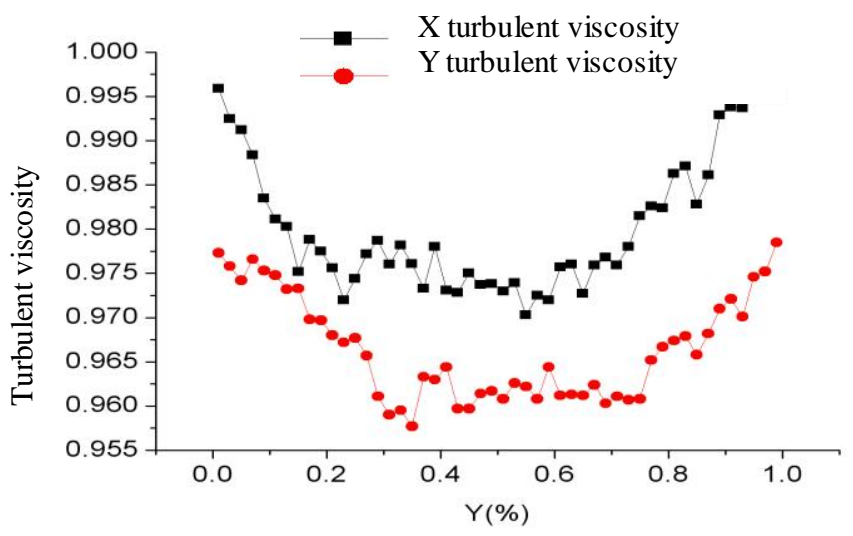

Figure 3: Molecular viscosity at diferent direction

Higher speed flow field particle clusters exist, and forms the disturbance source. Near-field velocity pulse jet boundary relative to the mainstream pulse of small, the $\mathrm{X}$ acceleration value is fluctuating around zero; main part is zero (fig. 4). At central pipe, the axial velocity fluctuation significantly, the $\mathrm{Y}$ acceleration value displays small fluctuations; $\mathrm{X}$ acceleration value is a few larger Speed particles clusters still exist. Far-field acceleration value is almost zero. The $\mathrm{Y}$ velocity distribution at $10 d$ place from nozzles is normal (compared with the distribution of the traditional number), but still with small fluctuations. At the both sides of axis, the velocity distribution and instability changes largely by few big-speed particles, the overall is symmetry. The negative velocity display back flow and entrainment.

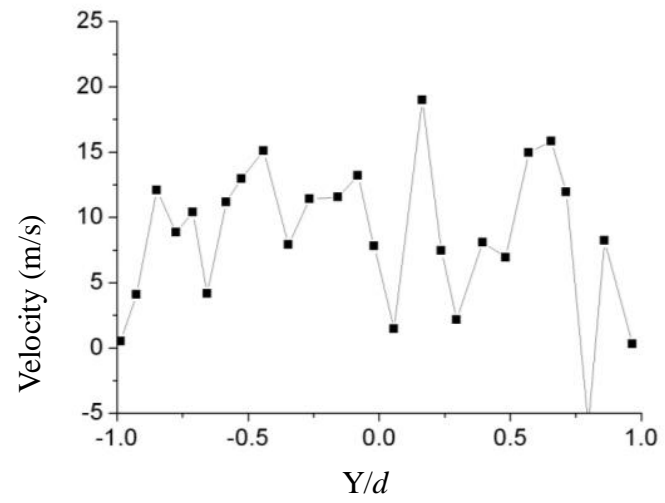

a) Shaft velocity at tube region

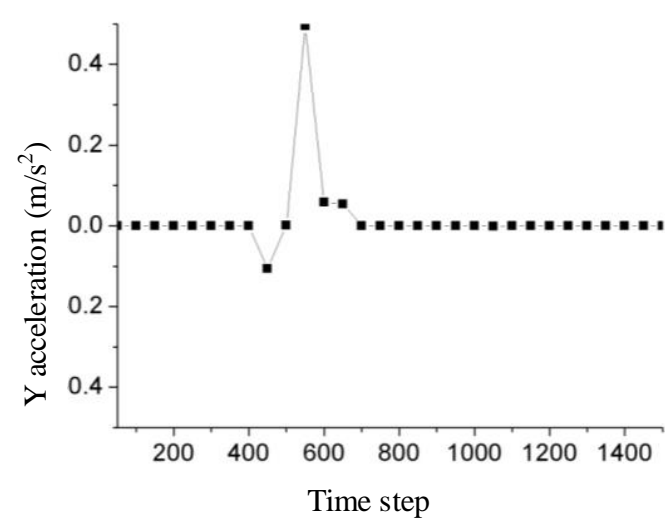

b) Shaft acceleration at tube region

Figure 4: Flow distributions diagram in tube region in case 1

Three programs calculate the Lagrange density can better reflect $L D$ values (as in fig. 5); the fourth option cannot be calculated. In case 1, The first and third option schemes value calculated is lower than that of the second option, but in case 2 , the first two calculation values are less than that of the third calculation 
value.

In case 1, the situation is different from that in the case 2. Considering the wall interactions, three kinds of $S P L$ are 133,133 and $126.4 \mathrm{~dB}$ respectively (fig. 6). At the $10 d$ and $5 d$ distances in case 2, the sound pressure calculated are $107.6,107.6 ; 115.5 \mathrm{~dB} 115.6 ; 115.6,117 \mathrm{~dB}$ respectively. The corresponding attenuations are $1.6,1.6$ and 0.3 decibels per pipe diameter.

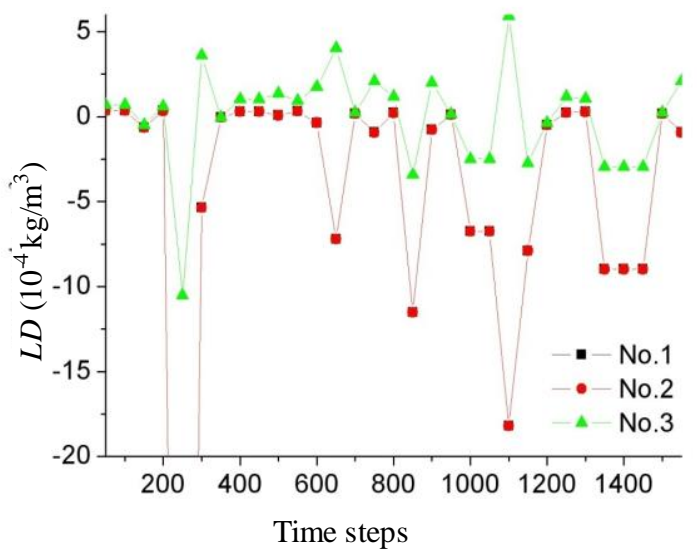

Figure 5: $L D$ comparison at the $10 d$ place axis in case 1

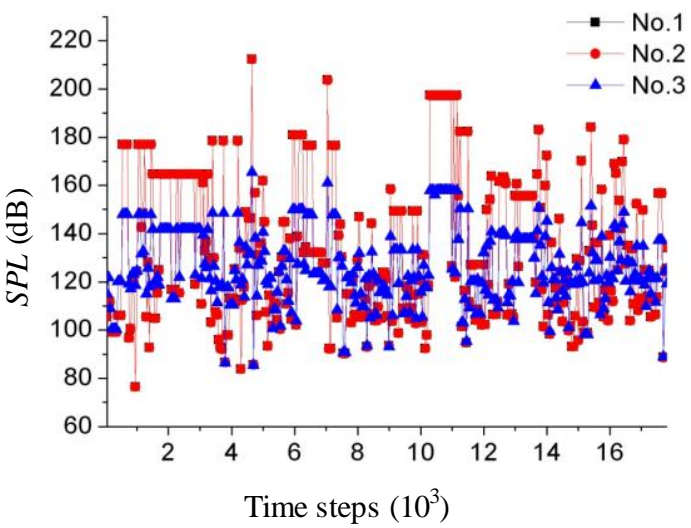

Figure 6: The $S P L$ values at the nozzle in case 2

With time step increasing, the density value variation in case 1 shows the two short intervals characteristics firstly, followed by another two large intervals (fig. 7). Further, intervals distance increases, but there exist two basic cycles. This is similar to frequency, and may reflect the inherent characteristics of the jet system.

At the tube monitoring point near the tube wall, the fluid particles collide with the tube wall frequently and randomly. This is the random effects results. The tube wall near the nozzle is the basic source of the jet sound, and it may be the dipole sound source (as in fig. 8).

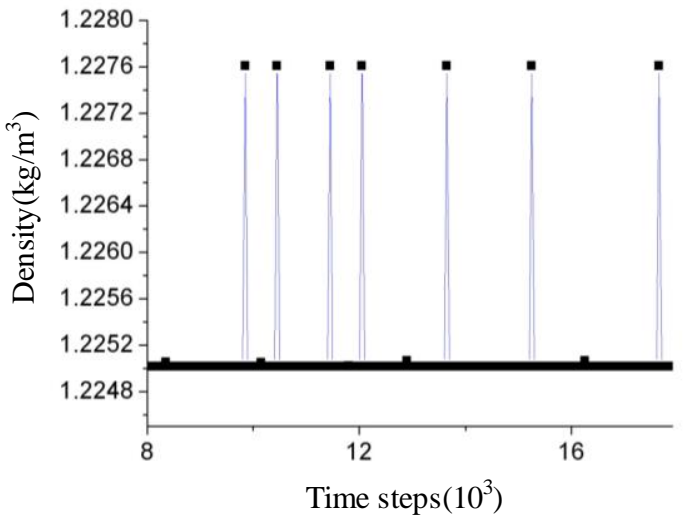

Figure 7: The density change at the $5 \mathrm{~d}$ place of in case 1

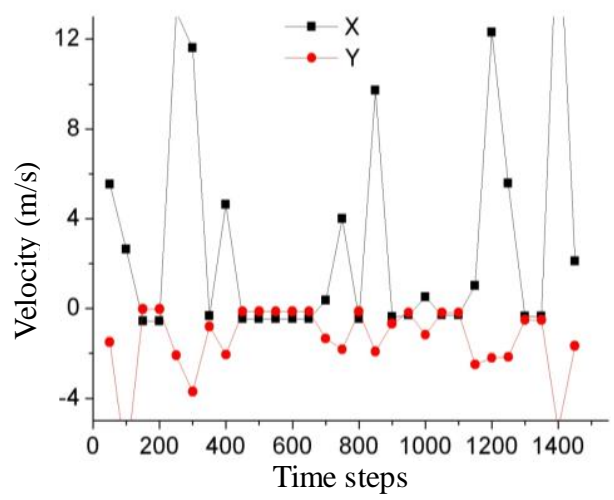

Figure 8: The internal wall speed of a control in case 2

\section{CONCLUSIONS}

In this paper, the jet flow field of two-dimensional micro-scale simulation of molecular dynamic, using the Lagrange density mechanism, describes the sound propagation. The main are as bellow:

Compared to the traditional theory, the results compare well with the literature [ㄷ]. This shows the 
nature of turbulence at all regions, velocity fluctuations displays out random features. The viscosity values are smaller in center region, but bigger at wall aside, no longer a constant.

Lagrange density can be convenient to calculate by the local density and pressure in order to obtain sound pressure, and the traditional definition style cannot get sound pressure values. The sound pressure values are similar to pulse pressure value, and Lagrange densities have two period times characteristics approximately.

At the jet flow field and pipes region, particles velocities distributions are consistent with LBM, but with many small groups which have bigger velocity, and form the disturbance sources.

The strong interaction with the tube wall produces greater sound pressure, such as dipole. Thus random collisions method at the tube wall is effective to deal with sound propagation problem.

Compared with the direct algorithm verlet algorithm is easier to load, effective to control temperature, and of the stalemate to Lagrange conservative force field nature. Molecular Mechanics programming can be achieved over the use of the space accuracy $10^{-8} \mathrm{~m}$ and the accuracy time $10^{-12} \mathrm{~s}$, which greatly improved the accuracy of the sound field simulation.

This paper offers preliminary basis and calculated basis for the molecular and macroscopic quantum aerodynamic problems.

\section{ACKNOWLEDGEMENT}

The authors wish to show our special thanks to the supports by the projects from Natural Science Foundation of China (No. 51376077, No. 50976044), Henan Higher Education Department Project for the excellent youth scholars in China (No. 2011GGJS-176), and Henan Province Education Department Project for the fundamental and advanced research in China (No. 14B470019).

\section{BIBLIOGRAPHY}

[1] Guo, Z. L., Zheng, C. G., Theory and Applications of Lattice Boltzmann Method, 1 ed., Science Press, Beijing, China, 2009.

[2] Succi, S., Lattice Boltzmann Equation for Fluid Dynamics and Beyond, 1 ed., Oxford Clarendon Press, London, England, 2001.

[3] Andrew, R. L., Molecular Modeling Principles and Application, 1 ed., Henry Ling Ltd., Dorchester, England, 2001.

[4] Frenkel, D., Smit, B., Understanding Molecular Simulation: From Algorithms to Applications, 1 ed., Academic Press, San Diego, USA, 2002.

[5] Stromberg, J. L., et al., "Flow Field and Acoustic Properties of Mach Number 0.9 Jet at A Low Reynolds Number", J. of Sound and Vib., v. 72, , pp. 159-163, 1980.

[6] Galassi, G., Tildesley, D. J., "Phase Diagrams of Diatomic Molecules: Using the Gibbs Ensemble Monte Carlo Method", Molecular Simulations, v. 11, n. 13, pp. 11-24, 1993.

[7] Coon, J. E., Gupta, S., et.el., "Isothermal Isobaric Molecular Dynamics Simulation of Diatomic Liquids and Their Mixtures", Chemical Physics, v. 113, n. 1, pp. 43-52, 1987.

[8] Delhommelle, J., Millie, P., "Inadequacy of the Lorentz-Berthelot Combing Rules for Accurate Predictions of Equilibrium Properties by Molecular Simulation”, Mol. Phys., v. 99, n. 8, pp. 619-625, 2001.

[9] Goldstein, M. E., Aeroacoustics, 1 ed., McGraw-Hill International Book Co., New York, USA, 1976. 